\title{
CAN FU ORIONIS OUTBURSTS REGULATE THE ROTATION RATES OF T TAURI STARS?
}

\author{
Robert Popham \\ Harvard-Smithsonian Center for Astrophysics
}

\begin{abstract}
We propose that FU Orionis outbursts may play an important role in maintaining the slow rotation of classical disk-accreting T Tauri stars. Current estimates for the frequency and duration of FU Orionis outbursts and the mass accretion rates of $\mathrm{T}$ Tauri and FU Orionis stars suggest that more mass may be accreted during the outbursts than during the T Tauri phases. If this is the case, then the outbursts should also dominate the accretion of angular momentum. During the outbursts, the accretion rate is so high that the magnetic field of the star should not disrupt the disk, and the disk will extend all the way in to the stellar surface. Standard thin disk models then predict that the star should accrete large amounts of angular momentum, which will produce a secular spinup of the star.

We present boundary layer solutions for FU Orionis parameters which show that the rotation rate of the accreting material reaches a maximum value which is far less than the Keplerian rotation rate at the stellar surface. This is due to the importance of radial pressure support at these high mass accretion rates. As a result, the rate at which the star accretes angular momentum from the disk drops rapidly as the stellar rotation rate increases. In fact, the angular momentum accretion rate drops below zero for stellar rotation rates which are always substantially below breakup, but depend on the mass accretion rate and on the adopted definition of the stellar radius. When the angular momentum accretion rate drops close to zero, the star will stop spinning up. Faster stellar rotation rates will produce negative angular momentum accretion which will spin the star down. Therefore, FU Orionis outbursts can keep the stellar rotation rate close to some equilibrium value for which the angular momentum accretion rate is small. We show that this equilibrium rotation rate may be similar to the observed rotation rates of $\mathrm{T}$ Tauri stars; thus we propose that FU Orionis outbursts may be responsible for the observed slow rotation of $\mathrm{T}$ Tauri stars. This mechanism is independent of whether the disk is disrupted by the stellar magnetic field during the T Tauri phase.
\end{abstract}


Subject headings: accretion, accretion disks - stars: formation-stars: pre-main-sequence - stars: rotation

\section{Introduction}

The FU Orionis stars are a class of accreting pre-main sequence stars which are observed to be $\mathrm{T}$ Tauri stars undergoing large outbursts (see Hartmann, Kenyon, \& Hartigan 1993 for a review). During the outbursts, these systems brighten by $\sim 5-6$ magnitudes over a period of $\sim 1-10$ years. They reach luminosities of a few hundred $\mathrm{L}_{\odot}$, with spectra indicating maximum temperatures $\sim 6500-7000 \mathrm{~K}$. The outbursts last for $\sim 100$ years, although this number is poorly known, since no outbursting systems have been observed to return to their pre-outburst state. Hartmann \& Kenyon $(1985,1987)$ showed that FU Orionis stars contain accretion disks by demonstrating that spectra of these systems contain double-peaked absorption lines. Simple steady accretion disk models of two FU Orionis systems, FU Orionis and V1057 Cygni, were constructed by Kenyon, Hartmann, \& Hewett (1988, hereafter KHH). The models which best fitted the observed broad-band spectral energy distributions of these systems had accretion rates $\dot{M} \sim 10^{-4} \mathrm{M}_{\odot} \mathrm{yr}^{-1}$, and central stars with masses $M_{*} \sim 0.3-1 \mathrm{M}_{\odot}$ and radii $R_{*} \sim 4 \mathrm{R}_{\odot}$. The presence of a disk in these systems suggests that their outbursts may result from a disk instability similar to those believed to cause similar outbursts in dwarf novae (Clarke, Lin, \& Papaloizou 1989; Clarke, Lin, \& Pringle 1990; Bell \& Lin 1994; Bell et al. 1995).

Disk accretion also has important implications for the rotational evolution of the accreting star. In the standard picture of disk accretion (Shakura \& Sunyaev 1973), the star accretes angular momentum from the disk. The angular momentum accretion rate is simply the Keplerian specific angular momentum at the stellar surface multiplied by the mass accretion rate. This quickly spins up the accreting star. Thus, in the standard picture, if T Tauri or FU Orionis stars have accreted even a small fraction of their mass through a disk, they should be spinning rapidly. The rotation rates of FU Orionis stars are not well known, since the accretion luminosity is so high that it overwhelms the stellar luminosity. The rotation rates of $\mathrm{T}$ Tauri stars are more easily observable and have been studied in some detail (Bouvier et al. 1993, 1995). They tend to be about a tenth of breakup speed, on average, with rotation periods usually around 6-8 days. Since the angular momentum added by disk accretion should rapidly spin $\mathrm{T}$ Tauri stars up to breakup speed, some authors have hypothesized that the spinup of $\mathrm{T}$ Tauri stars is controlled by the interaction between the disk and a strong stellar magnetic field (Königl 1991; Cameron \& Campbell 1993; Hartmann 1994; Shu et al. 1994). 
This mechanism for maintaining the spin rate of a star near some equilibrium value was first explored in detail in order to explain the spin behavior of some accreting neutron stars (Ghosh, Lamb, \& Pethick 1977; Ghosh \& Lamb 1979a,b). In this model, the stellar magnetic field disrupts the disk at some distance from the stellar surface. The location of this disruption radius is determined by the strength of the magnetic field and the mass accretion rate; a stronger magnetic field disrupts the disk farther away from the star, while a larger mass accretion rate moves the disruption radius closer to the stellar surface. The Keplerian rotation speed at the disruption radius determines approximately how fast the star will rotate. In order to produce the rotation speeds of most $\mathrm{T}$ Tauri stars, the disk would have to be disrupted around 5 stellar radii from the stellar surface, and for the accretion rates commonly ascribed to $\mathrm{T}$ Tauri stars, this would require a stellar magnetic field $\sim 1 \mathrm{kG}$ (Königl 1991).

The magnetic hypothesis for explaining the slow rotation of $\mathrm{T}$ Tauri stars does not include the effects of angular momentum accretion during FU Orionis outbursts. Event statistics of these outbursts, in which the number of observed events is compared to the number of young stars in the observable volume, suggest that each star probably undergoes multiple outbursts (Herbig 1977; Hartmann \& Kenyon 1985). Estimates for the time between outbursts vary widely from $\sim 10^{3}-10^{5}$ years (e.g. Bell \& Lin 1994; Kenyon 1995). If the accretion rate during this period is the typical $\mathrm{T}$ Tauri rate of $\sim 10^{-7} \mathrm{M}_{\odot} \mathrm{yr}^{-1}$, then $10^{-4}-10^{-2} \mathrm{M}_{\odot}$ will be accreted during the $\mathrm{T}$ Tauri phase. During the intervening FU Orionis outbursts, the accretion rate is $\sim 10^{-4} \mathrm{M}_{\odot} \mathrm{yr}^{-1}$ for $\sim 100$ years, so $\sim 10^{-2} \mathrm{M}_{\odot}$ should be accreted during the outburst. This suggests that as an accreting pre-main sequence star evolves through multiple cycles of FU Orionis outbursts and T Tauri quiescent phases, most of the mass accreted by the star may be added during the outbursts. If this is the case, then we should expect that the outbursts will also dominate the accretion of angular momentum.

During the FU Orionis outbursts, the stellar magnetic field should have little effect on the accretion disk. As noted above, in order to produce the observed rotation rates of $\mathrm{T}$ Tauri stars, the field should disrupt the disk at a radius of about 5 stellar radii. When such a star experiences an FU Orionis outburst, the mass accretion rate increases by a factor of order 1000. This should be sufficient to overwhelm the magnetic field, and allow the disk to reach all the way in to the stellar surface. In the standard picture of accretion disks, since the star accretes a large amount of mass during the FU Orionis outburst, it should also accrete a large amount of angular momentum, which will spin the star up substantially. This will in turn require that the star loses large amounts of angular momentum during the $\mathrm{T}$ Tauri phase in order to stay at the observed slow rotation rate. If the star accretes less mass in the T Tauri phase than it does in the FU Orionis outburst, it will be unable to lose 
sufficient angular momentum to spin back down, and over time, the star will continue to spin up toward breakup speed.

If the disk reaches the stellar surface, and the star is rotating slowly, a viscous boundary layer forms between the rapidly rotating disk material and the star. The boundary layer controls the flow of angular momentum between the disk and the star. Also, if the central star is not rotating, the boundary layer should produce as much luminosity as the disk. Clearly, if we wish to understand the angular momentum transfer and to compare disk models to observed spectra of FU Orionis systems, it is essential to include the boundary layer in our disk models.

Our initial investigation of the boundary layer structure examined the question of whether a star continues to accrete mass and angular momentum when it has been spun up to breakup speed (Popham \& Narayan 1991, hereafter PN91). We found that as the star approaches the breakup rotation rate, the amount of angular momentum accreted per unit of mass accreted drops dramatically, and can even become negative, so that the star continues to accrete mass while losing angular momentum to the disk. Thus, there is some equilibrium rotation rate close to breakup for which the star accretes no angular momentum, so that it can continue to accrete mass without spinning up or down. This study used a very simple disk model with a polytropic relation between the disk pressure and density, so it was not clear that similar conclusions would hold when a more realistic treatment of the disk was used. More recently, we have explored the structure of the boundary layer region for several types of accreting stars, including T Tauri and FU Orionis stars (Popham et al. 1993, hereafter PNHK) and cataclysmic variables (Narayan \& Popham 1993; Popham \& Narayan 1995), using a more realistic model which includes the energy balance and radiative transfer.

In this paper, we examine the angular momentum transfer between the star and the disk for boundary layer solutions calculated for parameters corresponding to FU Orionis systems. The boundary layer model we use is similar to the one used in our studies of cataclysmic variables (Narayan \& Popham 1993; Popham \& Narayan 1995) and in our previous study of pre-main sequence stars (PNHK). We find that many of the conclusions reached by PN91 continue to apply when our more detailed model is applied to FU Orionis systems. When the stellar rotation rate is plotted against the height of the disk at the stellar surface, the solutions fall on multiple solution branches. We find that the angular momentum accretion rate drops as the stellar rotation rate increases, and that solutions exist for a wide range of angular momentum accretion rates, including negative values. There is an equilibrium stellar rotation rate for which the star can accrete mass without spinning up or down. The major difference from the PN91 results is that for these FU 
Orionis-type solutions, the equilibrium stellar rotation rate is well below breakup.

On the basis of these solutions, we propose that FU Orionis outbursts play an important role in the spin evolution of accreting pre-main sequence stars. The large amount of mass accreted during an outburst can change the stellar rotation rate substantially. If more mass is accreted during the FU Orionis outburst phases than during the intervening $\mathrm{T}$ Tauri phases, then the stellar rotation rate will stay close to the equilibrium rate established during the FU Orionis phase. Although this equilibrium rate is rather uncertain, we will show that for reasonable choices of parameters, it is comparable to the observed rotation rates of $\mathrm{T}$ Tauri stars. Thus, angular momentum transfer during the FU Orionis outbursts can explain the slow rotation of $\mathrm{T}$ Tauri stars without invoking strong stellar magnetic fields. If $\mathrm{T}$ Tauri stars have weak fields which allow the accretion disk to extend down to the surface of the star and spin up the star, negative angular momentum accretion during the FU Orionis outburst phases can keep the star spinning slowly. Alternatively, if T Tauri stars have strong magnetic fields which disrupt the disk, the FU Orionis phases will still dominate the spin evolution as long as they dominate the mass accretion.

In $\S 2$, we discuss the model used to calculate our boundary layer and disk solutions for FU Orionis parameters, and the input parameters for our solutions. We describe a typical solution in detail in $\S 3$. We demonstrate the presence of multiple solution branches, and show how the character of the solutions varies along those branches. We also show a solution which accretes mass without accreting angular momentum. Finally, we show how variations in the mass accretion rate affect the solutions and solution branches. In $\S 4$, we describe the choice of an additional condition which allows us to find a relation between the stellar rotation rate and the angular momentum accretion rate. We discuss the spin evolution of T Tauri/FU Orionis systems and the implications of our results in $\S 5$.

\section{Disk and Boundary Layer Model}

\subsection{Disk Equations}

The model we use to study the boundary layer uses a version of the "slim disk" equations developed by Paczyński and collaborators (Paczyński \& Bisnovatyi-Kogan 1981; Muchotrzeb \& Paczyński 1982). This model is similar to the models used in previous papers (Narayan \& Popham 1993; PNHK; Popham \& Narayan 1995), so we will only describe it qualitatively here, and refer the reader to those papers for more details.

The model assumes a steady state, so that the mass and angular momentum accretion

rates $\dot{M}$ and $\dot{J}$ are constant with radius. We use the slim disk versions of the angular 
momentum, radial momentum, and energy equations. Thus, we dispense with some of the simplifying assumptions made in the standard thin disk treatment (Shakura \& Sunyaev 1973). We include pressure support and acceleration in the radial momentum equation, so that the rotational velocity $\Omega$ is not required to equal the Keplerian value $\Omega_{K}$. We allow the angular momentum accretion rate $\dot{J}$ to deviate from the standard value $\dot{M} \Omega_{K}\left(R_{*}\right) R_{*}^{2}$ by a factor $j$, so that $\dot{J}=j \dot{M} \Omega_{K}\left(R_{*}\right) R_{*}^{2}$. Finally, we drop the assumption that the energy dissipated by viscosity is radiated locally at the same radius in the disk. Instead, we include terms for the radial transport of energy by radiation and by the accreting material itself. The radiative transfer scheme we use is the one described in PNHK.

The modified angular momentum, radial momentum, and energy equations, along with the radiative transfer equations, provide a set of differential equations which can be solved with appropriate boundary conditions. The most important of these are the conditions on the angular velocity and the radial radiative flux. The angular velocity $\Omega$ must match the stellar rotation rate $\Omega_{*}$ at $R_{*}$, and the Keplerian value $\Omega_{K}\left(R_{\text {out }}\right)$ at $R=R_{\text {out }}=100 R_{*}$. At $R_{*}$, there is an outward radiative flux characterized by an effective temperature $T_{*}$, which we take to be $5000 \mathrm{~K}$. This produces a luminosity of $\sim 10^{34} \mathrm{ergs} \mathrm{s}^{-1}$ entering the boundary layer from the star, which is a small fraction of the accretion luminosity $L_{a c c} \sim 10^{36} \mathrm{ergs} \mathrm{s}^{-1}$. The equations are solved using a relaxation method.

\subsection{Solution Parameters}

Within the framework of the disk and boundary layer model described above and in the aforementioned papers, a large number of solutions can be found. These solutions are distinguished by various choices of input parameters. For our models, these include the mass accretion rate $\dot{M}$, the angular momentum accretion rate $\dot{J}$, the stellar mass, radius, and rotation rate $M_{*}, R_{*}$, and $\Omega_{*}$, and the viscosity parameter $\alpha$. In the case of FU Orionis systems, none of these parameters are directly observable, so they must be estimated by using the best current models to interpret the available observations.

\subsection{1. $\dot{M}, M_{*}$, and $R_{*}$}

These are the standard accretion disk parameters which determine the disk luminosity and temperature. Observed spectra of FU Orionis and V1057 Cygni were fitted with simple accretion disk models by KHH. Although these models did not include the boundary layer, they allow us to estimate the basic disk parameters as follows. First, the infrared flux is 
produced primarily in regions of the disk which are fairly far from the stellar surface, so that the flux produced is insensitive to the value of $R_{*}$. We have calculated simple blackbody spectra of our disk and boundary layer solutions for various choices of $\dot{M}$ and $M_{*}$, and compared them to photometric data of V1057 Cygni. Fits to infrared photometry suggest that the combination $\dot{M} M_{*} \simeq 5 \times 10^{-5} \mathrm{M}_{\odot}{ }^{2} \mathrm{yr}^{-1}$. Our spectra and fits will be presented in a future paper (Popham et al. 1995).

Another constraint is provided by spectra of FU Orionis and V1057 Cygni, which show that the maximum effective temperature reached in the inner disk is around $7000 \mathrm{~K}$ (KHH; Kenyon et al. 1989). In the standard Shakura \& Sunyaev (1973) model, the disk reaches a maximum effective temperature $T_{\max }=0.287\left(G M_{*} \dot{M} / R_{*}^{3} \sigma\right)^{1 / 4}$ at $R=49 / 36 R_{*}$. $T_{\max } \propto R_{*}^{-3 / 4}$, so it provides a reasonable way of estimating $R_{*}$; however, the ShakuraSunyaev formulation does not include any luminosity from the boundary layer, so the true temperature of the inner disk will be higher than $T_{\text {max }}$. For $\dot{M} M_{*}=5 \times 10^{-5} \mathrm{M}_{\odot}{ }^{2} \mathrm{yr}^{-1}$, we find $T_{\text {max }} \simeq 6500 \mathrm{~K}$ for $R_{*}=3 \times 10^{11} \mathrm{~cm}=4.31 \mathrm{R}_{\odot}$. This value for $R_{*}$ is somewhat larger than the observational estimates for $\mathrm{T}$ Tauri star radii, such as those tabulated by Bouvier et al. (1995), where $R_{*} \simeq 1.5-2.5 \mathrm{R}_{\odot}$.

This combination of parameters gives an accretion luminosity $L_{a c c} \simeq$ $1.4 \times 10^{36} \mathrm{ergs} \mathrm{s}^{-1} \simeq 350 L_{\odot}$, which agrees with the bolometric luminosity estimates for most of the FU Orionis systems listed by Bell \& Lin (1994). In the solutions presented in this paper, we keep $M_{*}=0.5 \mathrm{M}_{\odot}$ constant, and vary $\dot{M}$ and $R_{*}$ so that the accretion luminosity remains nearly constant. The three sets of solutions have $\dot{M}=10^{-4.0}, 10^{-4.15}$, and $10^{-4.3} \mathrm{M}_{\odot} \mathrm{yr}^{-1}$, and $R_{*}=4 \times 10^{11}, 3 \times 10^{11}$, and $2.25 \times 10^{11} \mathrm{~cm}$, respectively.

\subsection{2. $\alpha$}

The viscosity parameter $\alpha$ is more difficult to determine. The most common way to do so is to try to estimate the viscous timescale in the disk by observing the disk outburst behavior. This assumes that the FU Orionis outbursts are produced by disk instabilities, or at least by some disturbance which dies away on the viscous timescale. Disk instability models of FU Orionis outbursts have found that small values of $\alpha \sim 10^{-3}-10^{-4}$ were required to reproduce the long timescales of observed outbursts (Clarke, Lin \& Pringle 1990; Bell \& Lin (1994); Bell et al. 1995). Unfortunately, these values of $\alpha$ lead to problems with the evolutionary timescales and gravitational stability of the disk (Bell et al. 1995). We have therefore adopted an intermediate value of $\alpha=0.01$ which we use for all of our models. 


\subsection{3. $\Omega_{*}$ and $j$}

These parameters are usually ignored in accretion disk studies, since in the standard Shakura \& Sunyaev-type model, the accreting star is assumed to be nonrotating, and the angular momentum accretion rate is just given by the Keplerian specific angular momentum at the stellar surface multiplied by the mass accretion rate. Our study treats the boundary layer region explicitly, so these assumptions are no longer necessary. We treat both the stellar rotation rate and the angular momentum accretion rate as free parameters for purposes of finding our solutions. Later, when we discuss the spin evolution of accreting pre-main-sequence stars in $\S 4$, we will attempt to define a relation which specifies the angular momentum accretion rate as a function of the stellar rotation rate.

\section{Solutions and Solution Branches}

\subsection{A Typical Solution}

We begin by discussing an individual boundary layer solution with $\dot{M}=10^{-4.15} \mathrm{M}_{\odot} \mathrm{yr}^{-1}$ and $R_{*}=3 \times 10^{11} \mathrm{~cm}$, with $j=0.90$ and $H\left(R_{*}\right)=10^{11} \mathrm{~cm}$. These parameters are typical of most of the solutions presented here. Note that we have specified the disk height at $R_{*}$ rather than $\Omega_{*}$; the reason for this is discussed below. Figure 1 shows the variation of the rotational velocity $\Omega$, the radial velocity $v_{R}$, the effective temperature $T_{\text {eff }}$, and the disk height $H$ in the region $R=1-3 R_{*}$.

The rotational velocity $\Omega$ reaches a maximum around $R \simeq 5 \times 10^{11} \mathrm{~cm} \simeq 5 / 3 R_{*}$, so the dynamical boundary layer is about $2 / 3 R_{*}$ wide. Note that the maximum $\Omega$ is only about $70 \%$ of $\Omega_{K}$ at that radius. This means that even at the outer edge of the dynamical boundary layer, $\Omega^{2} R$ is only about $50 \%$ of the gravitational term $\Omega_{K}^{2} R$; the other half of the support against gravity is provided by the pressure gradient.

The radial velocity $v_{R}$ shows a similar profile to $\Omega$. It reaches a maximum of $\sim 3 \times 10^{4} \mathrm{~cm} \mathrm{~s}^{-1}$ at $R \simeq 6 \times 10^{11} \mathrm{~cm} \simeq 2 R_{*}$. Thus, the accreting material moves radially through the boundary layer region in about 1 year. In the boundary layer, the radial velocity drops rapidly, reaching $\sim-2000 \mathrm{~cm} \mathrm{~s}^{-1}$ at the stellar surface.

The effective temperature $T_{\text {eff }}$ peaks closer to the stellar surface than either $\Omega$ or $v_{R}$. In fact, the maximum $T_{\text {eff }} \sim 8300 \mathrm{~K}$ is reached at $R=3.8 \times 10^{11} \mathrm{~cm}$, in the middle of the dynamical boundary layer. The radiative energy released in the dynamical boundary layer propagates out to larger radii, but most of it has been radiated from the disk surface inside $2 R_{*}$. Thus, the thermal boundary layer (the region of elevated effective temperature) is 
only slightly wider than the dynamical boundary layer.

The disk height $H$ is fairly large: the ratio $H / R$ is close to $1 / 3$ throughout the inner region. Note that in earlier studies of the boundary layer structure at lower accretion rates, we generally found that $H$ increased rapidly in the pressure-gradient supported region inside the dynamical boundary layer. Here, as noted above, the accreting material is already substantially pressure-gradient supported at the outer edge of the dynamical boundary layer, and no rapid increase in $H$ is seen at the innermost radii.

\subsection{Solution Branches}

One goal of this paper is to examine the variation in the boundary layer structure as the various system parameters discussed in $\$ 2.2$ change. We are particularly interested in the spin history of accreting pre-main sequence stars, so we focus on the variations produced by changes in the rotation rate of the star $\Omega_{*}$ and the angular momentum accretion rate $j$. Accordingly, we first varied $\Omega_{*}$ while keeping all of the other parameters constant, including $\dot{M}, M_{*}, R_{*}, \alpha$, and $j$. This produced an intriguing result. We found that for some of our solutions, as we increased $\Omega_{*}$, the disk height $H_{*} \equiv H\left(R_{*}\right)$ increased, while other solutions showed the opposite behavior: $H_{*}$ decreased as the star spun faster.

We continued to systematically increase and decrease $\Omega_{*}$, and plotted the values of $H_{*}$ in the resulting solutions as a function of $\Omega_{*}$. This revealed that the tracks traced out in the $\Omega_{*}-H_{*}$ plane connected together into a single curve, like the one shown in Figure 2. This curve has a Z shape; multiple solutions can be found for a single value of $\Omega_{*}$. These solutions have different values of the disk height $H_{*}$, and they are distinct solutions, despite the fact that they share the same values of all of the input parameters $\dot{M}, M_{*}, R_{*}, \alpha, \Omega_{*}$, and $j$. In fact, by specifying $H_{*}$ rather than $\Omega_{*}$, we can find a unique solution. The Z-shaped curve has three branches; on the middle branch, $H_{*}$ increases with increasing $\Omega_{*}$, while on the upper and lower branches, $H_{*}$ decreases with increasing $\Omega_{*}$. The existence of multiple solution branches for a single value of $j$ agrees with the findings of our earlier work (PN91), which used a much simpler disk formulation.

\subsubsection{Variations Along the Branches}

The solutions along a Z-shaped curve show a wide range of variation in the structure of the boundary layer. In Figure 3 we have plotted 10 solutions with $H_{*}$ ranging from $8 \times 10^{10} \mathrm{~cm}$ to $1.25 \times 10^{11} \mathrm{~cm}$. The positions of these 10 solutions in the $\Omega_{*}-H_{*}$ plane are 
marked on the Z-shaped curve shown in Fig. 2 The solutions in the upper left have large values of $H_{*}$ and small values of $\Omega_{*}$ corresponding to a slowly rotating star. These solutions have a very broad boundary layer, as shown in Figure 3. The peak values of $\Omega$ and $v_{R}$ sit out at $R \simeq 4 R_{*}$. The peak effective temperature, occurs much closer to the star, but the region of elevated effective temperature extends out to several stellar radii. The disk height $H$ is approximately proportional to $R$, with $H / R \sim 0.4$, as shown in Fig. 3c.

Solutions with smaller values of $H_{*}$, but still lying on the upper branch, have progressively narrower boundary layers. The last solution shown on the upper branch, with $H_{*}=1.05 \times 10^{11} \mathrm{~cm}$, has $\Omega_{\max }$ at $R<2 R_{*}$ (Fig. 3). Meanwhile, $\Omega_{*}$ increases gradually,

reaching a value of $\sim 3.5 \times 10^{-6} \mathrm{~s}^{-1}$ at the transition from the upper to the middle branch. Note that this value of $\Omega_{*}$ is less than $10 \%$ of $\Omega_{K}\left(R_{*}\right) \simeq 5 \times 10^{-5} \mathrm{~s}^{-1}$.

As $H_{*}$ continues to decrease along the middle branch, the boundary layer continues to shrink in radial extent. The changes in the solutions along the middle branch with decreasing $H_{*}$ seem to continue smoothly the changes along the upper branch, with one exception: $\Omega_{*}$ drops back down toward zero. The $H_{*}=9 \times 10^{10} \mathrm{~cm}$ solution, near the transition from the middle to the lower branch, has $\Omega_{\max }$ at $R \simeq 4 \times 10^{11} \mathrm{~cm} \simeq 4 / 3 R_{*}$ (Fig. 3 ), so the radial extent of the boundary layer has decreased substantially. This solution has $\Omega_{*} \simeq 5 \times 10^{-7} \mathrm{~s}^{-1} \simeq 0.01 \Omega_{K}\left(R_{*}\right)$.

Finally, along the lower branch, the trends noted above for the upper and middle branches continue. The boundary layer becomes quite narrow, with $\Omega_{\max }$ at $R \simeq 1.15 R_{*}$ in the $H_{*}=8 \times 10^{10} \mathrm{~cm}$ solution. The value of $\Omega_{*}$ increases rapidly, and the solutions have a large angular velocity gradient $d \Omega / d R$ at $R_{*}$. This concentrated energy release produces a strong peak in $T_{\text {eff }}$ near $R_{*}$, but $T_{\text {eff }}$ begins to drop as $H_{*}$ continues to decrease, because the large values of $\Omega_{*}$ for these solutions means that a large portion of the rotational energy of the accreting material is retained rather than being dissipated in the boundary layer. The ratio $H / R$ varies more with $R$ for the lower branch solutions than for solutions on the other branches; the $H_{*}=8 \times 10^{10} \mathrm{~cm}$ solution has $H / R=0.24$ at $R=R_{*}$ but $H / R \simeq 1 / 3$ at $R=3 R_{*}$.

\subsection{Angular Momentum Accretion Rate}

Thus far we have kept the angular momentum accretion rate $j$ constant and showed that a family of solutions with a given value of $j$ traces out a Z-shaped curve in the $\Omega_{*}-H_{*}$ plane. Now we show the effects of varying the angular momentum accretion rate $j$. For each value of $j$, and using the same $\dot{M}, M_{*}, R_{*}$, and $\alpha$ as before, we can follow the variations in 
$\Omega_{*}$ as we vary $H_{*}$. These variations are shown in Figure 4.

As we increase $j$, we find a similar Z-shaped curve in the $\Omega_{*}-H_{*}$ plane, but the curve is displaced to the left, i.e. to smaller values of $\Omega_{*}$. For $j=0.91$, the transition between the lower and middle branches has moved to negative values of $\Omega_{*}$. The middle-to-upper branch transition also moves left, but by a smaller amount. The Z-shaped curves for adjacent values of $j$ nest inside one another, and they continue to be displaced to smaller $\Omega_{*}$ as $j$ continues to increase.

If we decrease $j$, the curves are displaced to larger $\Omega_{*}$, but they also begin to change shape dramatically. At $j=0.89$, the middle branch has become much shorter and steeper, so that it extends over a very narrow range in $\Omega_{*}$. At $j=0.84$, the middle branch has vanished completely, leaving a smooth, monotonic curve with $H_{*}$ decreasing as $\Omega_{*}$ increases.

If we continue to decrease $j$, we continue to find solutions, even for $j \leq 0$. In these solutions, the star accretes mass while losing angular momentum. An example of a $j=0$ solution is shown in Figure 5. The angular velocity continues to rise all the way in to the stellar surface. Nonetheless, $\Omega_{*}$ is far below $\Omega_{K}\left(R_{*}\right)$, so the inner region of the disk is almost completely pressure-supported. There is no maximum in $\Omega$, so in essence there is no boundary layer at all. The disk is fairly thick, with $H / R \simeq 0.4$. We can find solutions for negative values of $j$, and even for large negative values such as $j=-10$. As $j$ drops, the disk continues to thicken. We have included the curves for $j=0$ and $j=-1$ in the $\Omega_{*}-H_{*}$ plane in Figure 4. The curves are rather similar to the $j=0.84$ curve discussed above; $H_{*}$ decreases monotonically with increasing $\Omega_{*}$.

\subsection{Variations with $\dot{M}$}

Having described the appearance of the family of curves in the $\Omega_{*}-H_{*}$ plane produced by a range of values of $j$, we can examine the variations in this family of curves as the mass accretion rate changes. As discussed in $\S 2.2$, we also vary $R_{*}$ along with $\dot{M}$ so as to keep the accretion luminosity approximately constant, so that we examine 3 combinations of $\dot{M}$ and $R_{*}: \dot{M}=10^{-4.0}, 10^{-4.15}$, and $10^{-4.3} \mathrm{M}_{\odot} \mathrm{yr}^{-1}$, and $R_{*}=4 \times 10^{11}, 3 \times 10^{11}$, and $2.25 \times 10^{11} \mathrm{~cm}$, respectively.

In Figure 6, we show the families of curves in the $\Omega_{*}-H_{*}$ plane for $\dot{M}=10^{-4.0} \mathrm{M}_{\odot} \mathrm{yr}^{-1}$, $R_{*}=4 \times 10^{11} \mathrm{~cm}$ and for $\dot{M}=10^{-4.3} \mathrm{M}_{\odot} \mathrm{yr}^{-1}, R_{*}=2.25 \times 10^{11} \mathrm{~cm}$. These can be compared to Fig. 4, which used intermediate values of $\dot{M}=10^{-4.15} \mathrm{M}_{\odot} \mathrm{yr}^{-1}, R_{*}=3 \times 10^{11} \mathrm{~cm}$. The shapes of the curves and their variation with $j$ are quite similar for the 3 different accretion rates. The major difference is that the family of curves is displaced to larger $\Omega_{*}$ 
as $\dot{M}$ decreases, and to smaller $\Omega_{*}$ as $\dot{M}$ increases. Thus, at the lowest accretion rate, $\dot{M}=10^{-4.3} \mathrm{M}_{\odot} \mathrm{yr}^{-1}$, the transition from Z-shaped to monotonic curves occurs around $\Omega_{*} \simeq 1.5 \times 10^{-5} \mathrm{~s}^{-1} \simeq 0.2 \Omega_{K}\left(R_{*}\right)$. This can be compared to the $\dot{M}=10^{-4.15} \mathrm{M}_{\odot} \mathrm{yr}^{-1}$ case, where the same transition happens around $\Omega_{*} \simeq 5 \times 10^{-6} \mathrm{~s}^{-1} \simeq 0.1 \Omega_{K}\left(R_{*}\right)$. At $\dot{M}=10^{-4.0} \mathrm{M}_{\odot} \mathrm{yr}^{-1}$, all of the Z-shaped curves have moved to $\Omega_{*}<0$, corresponding to a star rotating in the opposite sense to the disk.

\section{Spin Evolution of Pre-Main-Sequence Stars}

\subsection{Angular Momentum Accretion}

In order to study the spin evolution of a disk-accreting star, we need to know how much angular momentum is accreted by the star as a function of its rotation rate. The answer to this question will determine the spin evolution of the star. Of course, the spin evolution may be complicated by variations in the mass accretion rate or the mass and radius of the star. These considerations are important for T Tauri/FU Orionis stars, and we will discuss them later.

To begin with, however, we concentrate on the variation of $j$ as a function of $\Omega_{*}$ for a star with constant $\dot{M}, M_{*}$, and $R_{*}$. The general sense of this variation is clear from Figs. 4 and 6 , which show how $j$ varies with $\Omega_{*}$ and $H_{*}$. If we assume for the moment that $H_{*}$ stays constant, then $j$ decreases as $\Omega_{*}$ increases. At small values of $\Omega_{*}$, where the constant- $j$ curves are Z-shaped, $j$ decreases gradually. As the star spins up, the constant- $j$ curves become monotonic, and $j$ decreases more rapidly. This suggests that the spin evolution of the star proceeds as follows: as long as $j$ remains positive, the star continues to spin up. Eventually, $j$ decreases to zero, and the star reaches an equilibrium rotation rate where it neither spins up nor spins down.

The major uncertainty in this picture is in $H_{*}$ : for a given value of $\Omega_{*}, j$ also depends strongly on the value of $H_{*}$. Thus, in some regions of the $\Omega_{*}-H_{*}$ plane, if $H_{*}$ were to decrease rapidly enough as $\Omega_{*}$ increases, $j$ would increase. As we discussed in $\S 3$, we can find solutions by specifying $j$ and either $\Omega_{*}$ or $H_{*}$. In nature, one would expect that only the stellar rotation rate $\Omega_{*}$ is an intrinsic parameter of the accreting system, and that both $j$ and $H_{*}$ should be determined by the physics of the accretion flow. In order to reproduce this situation within our model, we need an additional condition which gives $H_{*}$ as a function of $\Omega_{*}$. Such a condition will define a track in the $\Omega_{*}-H_{*}$ plane, and the variation of $j$ as a function of $\Omega_{*}$ along this track provides a basis for understanding the spin evolution of accreting stars. 


\subsection{Need for an Additional Boundary Condition}

The major reason for the uncertainty in $H_{*}$ is the lack of a clear definition of the stellar radius $R_{*}$. In the solutions presented thus far, we have specified a value for $R_{*}$, but we have not specified a boundary condition which ensures that the specified $R_{*}$ represents the true stellar radius in any physical sense. As a result, we can obtain a wide range of solutions which have very different physical characteristics at $R_{*}$, as shown in Fig. 3. Thus, we need an additional boundary condition, a definition for $R_{*}$ which marks the location of the transition from the boundary layer to the star, based on the changes in the accretion flow.

This is a difficult task, for several reasons. First, our model is set up in a way that specifically tries to avoid making distinctions between the disk, boundary layer and star. All of these regions are assumed to obey the same equations, with the same physical parameters. Second, the distinction between star and disk is much more difficult to make at large accretion rates. This can be seen clearly in Fig. 1. It is quite difficult to separate the accretion flow into different regions; $\Omega, T_{\text {eff }}$ and $H$ all vary smoothly over large length scales. This can be contrasted with the clear differences between the disk, boundary layer, and star which are visible in models for lower accretion rates, like those for cataclysmic variables (Popham \& Narayan 1995) and T Tauri stars (PNHK). In those models, $\Omega$ dropped rapidly in the boundary layer, and then stayed almost constant in the star; the transition between the two regions was quite rapid. Similarly, $H$ began to rise rapidly with decreasing $R$ inside the boundary layer. In the current solutions, the $\Omega$ profile shows only weak indications of leveling off, and $H$ continues to decrease approximately linearly with $R$. This makes it quite difficult to select a radius which represents a transition from boundary layer to star.

Another perspective on this issue comes from examining the range of solutions in Fig. 3 , all of which use a single value of $j$. One way to locate the stellar radius is to select a solution from this set for which the boundary layer-star transition appears to occur at the inner edge of our computational grid, where $R=R_{*}$. It seems clear that the solutions with the smallest values of $H_{*}$ are not appropriate. They have very large values of $d \Omega / d R$ at $R_{*}$, and it seems clear that if the solution were to continue in to $R<R_{*}$, $\Omega$ would continue to drop precipitously. Thus, it appears that in these solutions, $R_{*}$ falls in the middle of the boundary layer, and the boundary layer-star transition would occur at $R<R_{*}$. On the other hand, the solutions with the largest values of $H_{*}$ have very broad regions, extending

out to a few times $R_{*}$, in which $\Omega$ decreases quite gradually. It appears that these solutions have reached the boundary layer-star transition at $R>R_{*}$, and the material then simply continues to settle as it flows in slowly to $R_{*}$. The intermediate solutions with $H_{*} \simeq 10^{11} \mathrm{~cm}$ seem to have the best characteristics. Both $\Omega$ and $v_{R}$ have dropped substantially but are 
leveling off around $R_{*}$.

\subsection{Choice of a Boundary Condition}

We have many possible choices for boundary conditions to specify $R_{*}$. One possibility would be to try to match the shape of the disk surface to the shape of the surface of a star rotating at a speed $\Omega_{*}$; however, as we have discussed, the disk height $H$ does not vary in a way that resembles the transition from a disk to a star, so this would be difficult. The best variables to use for a boundary condition are probably the angular and radial velocities $\Omega$ and $v_{R}$. These variables show directly where the boundary layer is located, and compared to other variables like the central and effective temperatures of the disk, they depend far less on the details of radiative transfer, opacities, etc.

Of the two velocities, we believe that the radial velocity provides a superior measure of the location of the boundary layer-star transition. This is because the angular velocity may or may not drop as the accreting material approaches the stellar surface; if the star is rapidly rotating, $\Omega$ may drop only slightly, or may continue to increase, as we saw in the solutions with small and negative values of $j$ in $§ 3.3$. In such cases, it becomes very difficult to distinguish the disk from the star on the basis of the $\Omega$ profile. The radial velocity, on the other hand, always reaches a maximum value and then drops substantially as it approaches the stellar surface. Put another way, $\Omega_{*}$ does not have to be small, but $v_{R, *}$ does.

The simplest condition we can put on $v_{R}$ is simply to select a value for $v_{R, *}$, and define $R_{*}$ as the radius where $v_{R}=v_{R, *}$. This can then be used to determine the variation of $H_{*}$ and $j$ with $\Omega_{*}$. One simple way to do this is to keep $j$ constant and vary $H_{*}$, so as to move along one of the constant- $j$ curves shown in Figs. 4 and 6. Fig. 3 shows that $v_{R}$ varies monotonically along a constant- $j$ curve, such that $v_{R}$ decreases with increasing $H_{*}$. Thus, by varying $H_{*}$, we can find a solution for which $v_{R}=v_{R, *}$ at $R=R_{*}$. Corresponding

solutions can be found for other values of $j$, and the values of $\Omega_{*}$ and $H_{*}$ for these solutions will then produce the desired relations for $H_{*}\left(\Omega_{*}\right)$ and $j\left(\Omega_{*}\right)$.

\subsection{Variation of $j$ with $\Omega_{*}$}

In order to illustrate how $j$ varies with $\Omega_{*}$ for this boundary condition, we choose a typical value of $v_{R, *}=1000 \mathrm{~cm} \mathrm{~s}^{-1}$. This value is a factor of $\sim 2 \times 10^{4}$ smaller than the free-fall velocity, and it is $5-15 \%$ of the maximum radial velocity reached in the resulting solutions. The variation of $H_{*}\left(\Omega_{*}\right)$ for the solutions with $v_{R}=v_{R, *}$ at $R_{*}=3 \times 10^{11} \mathrm{~cm}$ 
is shown as a dashed line in Fig. 4. Note that $H_{*}$ is nearly constant for the full range of $\Omega_{*}$, with $H_{*} \simeq 1.07-1.11 \times 10^{11} \mathrm{~cm}$, so that $H_{*} / R_{*} \simeq 0.36$. There is some variation of $H_{*}$; it decreases gradually from about $1.088 \times 10^{11} \mathrm{~cm}$ at $\Omega_{*}=0$ to $1.075 \times 10^{11} \mathrm{~cm}$ at $\Omega_{*} \simeq 1.3 \times 10^{-5} \mathrm{~s}^{-1}$, and then rises more rapidly, reaching about $1.108 \times 10^{11} \mathrm{~cm}$ at $\Omega_{*} \simeq 1.83 \times 10^{-5} \mathrm{~s}^{-1}$.

More importantly, $j$ decreases with increasing $\Omega_{*}$; as the star spins up, it accretes less angular momentum. Figure 7 a shows the variation of $j$ as a function of $\Omega_{*} ; j$ decreases gradually at first, and then drops more rapidly. The angular momentum accretion rate reaches zero at $\Omega_{*} \simeq 1.5 \times 10^{-5} \mathrm{~s}^{-1}$, and rapidly drops to $j=-1$ at $\Omega_{*} \simeq 1.8 \times 10^{-5} \mathrm{~s}^{-1}$. Note that these values of $\Omega_{*}$ are only about one third of the Keplerian angular velocity at this radius, $\Omega_{K}\left(R_{*}\right) \simeq 5 \times 10^{-5} \mathrm{~s}^{-1}$. Thus, the star stops accreting angular momentum when it is still rotating well below breakup.

At other mass accretion rates, the variation of $H_{*}$ and $j$ with increasing $\Omega_{*}$ is quite similar: $H_{*}$ stays approximately constant and $j$ decreases gradually at first, then more rapidly. The major difference is that $j$ decreases more rapidly at higher mass accretion rates, and reaches $j=0$ at a smaller value of $\Omega_{*}$. This is illustrated in Figure $7 \mathrm{~b}$ and c, where we plot $j\left(\Omega_{*}\right)$ for the $\dot{M}=10^{-4.0} \mathrm{M}_{\odot} \mathrm{yr}^{-1}$ and $\dot{M}=10^{-4.3} \mathrm{M}_{\odot} \mathrm{yr}^{-1}$ solutions. We have used the same criterion, $v_{R, *}=-1000 \mathrm{~cm} \mathrm{~s}^{-1}$, to define the stellar radius. At $\dot{M}=10^{-4.0} \mathrm{M}_{\odot} \mathrm{yr}^{-1}, j=0$ for $\Omega_{*} \simeq 6.6 \times 10^{-6} \mathrm{~s}^{-1}$, corresponding to a rotation period of $\sim 11$ days. Also note that for this higher accretion rate, $j$ is substantially smaller than one even at $\Omega_{*}=0$. At $\dot{M}=10^{-4.3} \mathrm{M}_{\odot} \mathrm{yr}^{-1}, \Omega_{*}(j=0) \simeq 3 \times 10^{-5} \mathrm{~s}^{-1}$, which corresponds to a much shorter rotation period of $\sim 2.4$ days. Recall that as $\dot{M}$ changes, we have also varied $R_{*}$ by a similar amount in order to keep the accretion luminosity constant. Thus the variation in $\Omega_{*}(j=0)$ as a fraction of the breakup rotation rate $\Omega_{K}\left(R_{*}\right)$ is not as dramatic as the variation in $\Omega_{*}(j=0)$ itself; we find $\Omega_{*}(j=0) / \Omega_{K}\left(R_{*}\right) \simeq 0.2,0.3,0.4$ for $\dot{M}=10^{-4.0}, 10^{-4.15}, 10^{-4.3} \mathrm{M}_{\odot} \mathrm{yr}^{-1}$, respectively.

\section{Discussion}

\subsection{Spin Evolution of T Tauri/FU Orionis Stars}

We have found that boundary layer solutions for parameters corresponding to FU Orionis outbursts have small and negative angular momentum accretion rates for fairly low stellar rotation rates. This suggests that angular momentum transfer during the FU Orionis outbursts can keep the rotation rate of T Tauri/FU Orionis stars near to an equilibrium rotation rate $\Omega_{*, e q, F U} \simeq \Omega_{*}(j=0)$. If the star is rotating faster than $\Omega_{*, e q, F U}$ when it 
experiences an FU Orionis outburst, it will lose angular momentum in the outburst phase and spin back down to $\Omega_{*, e q, F U}$. If, on the other hand, the star is rotating slowly when an outburst occurs, it will have $j \sim 1$ in the outburst phase and will spin up toward $\Omega_{*, e q, F U}$. We have found that for accretion rates $\dot{M}=5 \times 10^{-5}-10^{-4} \mathrm{M}_{\odot} \mathrm{yr}^{-1}, j=0$ for spin rates $\Omega_{*}(j=0) \simeq 0.66-3.04 \times 10^{-5} \mathrm{~s}^{-1} \simeq \Omega_{*, e q, F U}$, corresponding to rotation periods of $2.4-11$ days. These are similar to the observed rotation periods of $\mathrm{T}$ Tauri stars (Bouvier et al. 1993, 1995); however, $\Omega_{*}(j=0)$ is sensitive to the value assumed for $v_{R, *}$, as discussed below.

During the T Tauri phase, the stellar rotation rate will tend to move toward a different equilibrium value $\Omega_{*, e q, T T}$. If the stellar magnetic field is too weak to disrupt the disk, then the star will spin up with $j \sim 1$ until it reaches $\Omega_{*, e q, T T} \simeq \Omega_{K}\left(R_{*}\right)$. If the stellar field does disrupt the disk, then $\Omega_{*, e q, T T}$ will be determined by the radius where the disruption occurs, and may be smaller or larger than $\Omega_{*, e q, F U}$. Therefore $j$ may be positive or negative, with a magnitude $|j| \lesssim\left(R_{d} / R_{*}\right)^{1 / 2}$ (Ghosh, Lamb, \& Pethick 1977), where $R_{d}$ is the radius at which the field disrupts the disk. Models of magnetically disrupted disks in T Tauri stars suggest $R_{d} \sim 4-5 R_{*}$, which would give $j \sim \pm 2$.

The spin evolution of a T Tauri/FU Orionis star will depend on $\Omega_{*, e q, F U}, \Omega_{*, e q, T T}$, and on the amounts of mass accreted in typical FU Orionis and T Tauri phases, $\Delta M_{F U}$ and $\Delta M_{T T}$. If $\Delta M_{F U}>\Delta M_{T T}$, as suggested by current estimates of the frequency and duration of FU Orionis outbursts, the stellar rotation rate $\Omega_{*}$ will move toward $\Omega_{*, e q, T T}$ during each $\mathrm{T}$ Tauri phase, but will return to $\Omega_{*, e q, F U}$ during each FU Orionis phase.

How large will the excursions in $\Omega_{*}$ be? A rigidly rotating star has angular momentum $J=I \Omega_{*}$, where $I$ is the star's moment of inertia, so $\dot{J}=\dot{I} \Omega_{*}+I \dot{\Omega}_{*}$. If we assume that the moment of inertia changes slowly, so that $\dot{J} \simeq I \dot{\Omega}_{*}$, then the star spins up and down at a rate $\dot{\Omega}_{*} / \Omega_{K}\left(R_{*}\right)=(j / k)(\dot{M} / M)$, where we have defined $I \equiv k M R_{*}^{2}$. Since $k$ is substantially less than 1 , the increase in $\Omega_{*}$ as a fraction of the breakup rotation rate $\Omega_{K}\left(R_{*}\right)$ is several times larger than the fractional increase in the stellar mass $M$. If we assume $j=1, k=0.2$, then the accretion of $\Delta M=0.01 \mathrm{M}_{\odot}$ onto a $0.5 \mathrm{M}_{\odot}$ star would increase $\Omega_{*}$ by $0.1 \Omega_{K}\left(R_{*}\right)$. If the typical change in $\Omega_{*}$ during the $\mathrm{T}$ Tauri phase is larger than the difference between the equilibrium spin rates $\Omega_{*, e q, F U}$ and $\Omega_{*, e q, T T}$, then $\Omega_{*}$ will reach $\Omega_{*, e q, T T}$ during each $\mathrm{T}$ Tauri phase, and remain there for the duration of the phase.

It is important to note that even though the FU Orionis phases may produce more mass accretion than the $\mathrm{T}$ Tauri phases, $\Delta M_{F U}>\Delta M_{T T}$, a $\mathrm{T}$ Tauri/FU Orionis star will still spend most of its lifetime with $\Omega_{*}$ moving away from $\Omega_{*, e q, F U}$, due to the short duration of the FU Orionis phases. This means that the mean value of $\Omega_{*}$ during the $\mathrm{T}$ Tauri phase will be offset somewhat from $\Omega_{*, e q, F U}$. The sign of this offset will depend on 
whether the star spins up or down during the T Tauri phase, i.e. on whether $\Omega_{*, e q, T T}$ is smaller or larger than $\Omega_{*, e q, F U}$. Also, the return to the T Tauri state after an FU Orionis outburst may be gradual, with the mass accretion rate declining slowly over a period of a few decades. At mass accretion rates intermediate between T Tauri and FU Orionis rates, the star should spin up, since the magnetic field may still be too weak to disrupt the disk, while the mechanism described in this paper will only operate at a large value of the equilibrium rotation rate. Despite these deviations, the high accretion rate of mass and angular momentum in the FU Orionis phase will return $\Omega_{*}$ to $\Omega_{*, e q, F U}$ quite rapidly, and as long as $\Delta M_{F U}>\Delta M_{T T}, \Omega_{*}$ will continue to be close to $\Omega_{*, e q, F U}$.

Note also that in general the equilibrium spin rate $\Omega_{*, e q}$ will differ slightly from the value of $\Omega_{*}$ which gives $j=0$. Since $\dot{J}=I \dot{\Omega}_{*}+\dot{I} \Omega_{*}$, we will have $\dot{\Omega}_{*}=0$ for $\dot{J}=\dot{I} \Omega_{*}$.

This simply means that in equilibrium, $\dot{J}$ must be sufficient to compensate for changes in the star's moment of inertia. If the moment of inertia increases in response to accretion, $j>0$ at $\Omega_{*, e q}$. If the moment of inertia decreases, as it might in an accreting white dwarf, then $j\left(\Omega_{*, e q}\right)<0$. For instance, if the star's radius of gyration stays constant, so that $\dot{I} / I=\dot{M} / M$, then $j\left(\Omega_{*, e q}\right)=k \Omega_{*, e q} / \Omega_{K}\left(R_{*}\right)$, where again $I \equiv k M R_{*}^{2}$. If $\Omega_{*, e q} / \Omega_{K}\left(R_{*}\right)$ is in the range 0.2-0.4 as suggested by our results, and $k \simeq 0.2$, then $j\left(\Omega_{*, e q}\right) \simeq 0.04-0.08$, and $\Omega_{*, e q}$ will be only slightly smaller than $\Omega_{*}(j=0)$.

\subsection{Solutions with Negative Angular Momentum Accretion}

One of the most important results of this paper is that it establishes the existence of disk and boundary layer solutions with small and negative angular momentum accretion rates. In these solutions, the central star accretes mass but loses angular momentum. This is quite different from the standard thin disk formulation, in which the angular momentum accretion rate is always $\dot{J}=\dot{M} \Omega_{K}\left(R_{*}\right) R_{*}^{2}$, or $j=1$ in our units. The key to understanding this result is that the $\Omega$ profiles of our solutions can be very different from the purely Keplerian $\Omega$ assumed in the thin disk case. Most importantly, $\Omega$ does not reach a maximum and then drop down to the stellar rotation rate $\Omega_{*}$. Instead, $\Omega$ continues to increase all the way in to the stellar surface at $R=R_{*}$. The fact that $\Omega$ is monotonic means that there is no radius $R_{\Omega_{m} a x}$ at which $d \Omega / d R=0$, where the viscous torque would vanish, and the angular momentum accretion rate would simply be the amount of angular momentum carried in the accreting material at that radius, $\dot{J}=\dot{M} \Omega_{\max } R_{\Omega_{m} a x}^{2}$. Since for a steady flow $\dot{J}$ must be constant with radius, this would constrain $\dot{J}$ to be positive. In our low- $j$ and negative- $j$ solutions, there is no maximum in $\Omega$, so that the viscous torque carries angular momentum outward at all radii. If the torque exceeds the rate at which angular momentum is carried 
in by the accreting material, the net angular momentum accretion rate will be negative.

These solutions are very similar to the ones we found in an earlier paper (PN91). In that paper, we calculated the structure of the boundary layer using a simplified disk model with a polytropic pressure-density relation. We found that our solutions formed two branches in the $\Omega_{*}-H_{*}$ plane for large values of $j \sim 1$. We did not find the lower branch, but seems likely that it exists for these solutions as well. The PN91 solution branches formed sharp corners where they met, unlike the rounded transitions between branches seen in the current solutions. This difference probably arises from the very different disk thicknesses in the two studies; the PN91 solutions generally had $H / R<0.01$. We were also able to find solutions with small and negative values of $j$; for these values only a single branch was present in the $\Omega_{*}-H_{*}$ plane. Thus, despite the use of a different disk model, the qualitative behavior of these solutions is very close to that of the solutions described in this paper.

One important question is why the solutions presented here reach negative angular momentum accretion rates when the stellar rotation rate $\Omega_{*}$ is still well below the breakup rate $\Omega_{K}\left(R_{*}\right)$. For the PN91 solutions, we defined the stellar radius as the point where $H / R=0.1$ in the accretion flow, and found that with this definition, $j$ was very close to 1 for most $\Omega_{*}$, but dropped precipitously when $\Omega_{*} \simeq 0.915 \Omega_{K}\left(R_{*}\right)$. We have seen that the current solutions reach $j=0$ for $\Omega_{*} \sim 0.2-0.5 \Omega_{K}\left(R_{*}\right)$. As discussed above, solutions with small or negative values of $j$ must have $d \Omega / d R<0$ for all $R$. Thus, if $\Omega \simeq \Omega_{K}$ close to $R_{*}$, the star must be rotating close to breakup, $\Omega_{*} \simeq \Omega_{K}\left(R_{*}\right)$, in order to allow a negative- $j$ solution. If $\Omega_{*}$ is much less than $\Omega_{K}\left(R_{*}\right), \Omega$ will have to decrease close to $R_{*}$, and there will be a maximum in $\Omega$, so that $j \simeq 1$.

In the FU Orionis solutions presented here, two factors combine to produce small and negative values of $j$ when $\Omega_{*} \ll \Omega_{K}\left(R_{*}\right)$. The first is the large radial width of the boundary layer. In the solutions with $j \sim 1, \Omega$ peaks fairly far from $R_{*}$, so that the radial width of the boundary layer is comparable to $R_{*}$. At the radius where $\Omega$ peaks, $\Omega_{K}$ is substantially smaller than $\Omega_{K}\left(R_{*}\right)$; for instance, at $R=2 R_{*}, \Omega_{K} \simeq 0.35 \Omega_{K}\left(R_{*}\right)$. As the star spins up, $\Omega_{*}$ only needs to reach this value to make $d \Omega / d R<0$ everywhere and allow $j$ to be small or negative. Second, pressure support plays an important role, and $\Omega$ is substantially smaller than $\Omega_{K}$. This allows solutions with $d \Omega / d R<0$ everywhere for even smaller values of $\Omega_{*}$. This also explains why we reach $j=0$ at smaller $\Omega_{*}$ for larger values of $\dot{M}$; as $\dot{M}$ increases, pressure support plays a larger role, the disk becomes thicker, and $\Omega$ is a smaller fraction of $\Omega_{K}$.

It is also worth noting that the total accretion luminosity increases substantially as $j$ 
decreases. In general, the accretion luminosity varies according to the expression

$$
L_{a c c} \simeq \frac{G M_{*} \dot{M}}{R_{*}}\left[1-j \frac{\Omega_{*}}{\Omega_{K}\left(R_{*}\right)}+\frac{1}{2} \frac{\Omega_{*}^{2}}{\Omega_{K}\left(R_{*}\right)^{2}}\right]
$$

(Popham \& Narayan 1995). Thus, for large negative values of $j$, the accretion luminosity can be substantially larger than the standard value $G M_{*} \dot{M} / R_{*}$. If the star spins up substantially during the T Tauri phase, then when an FU Orionis outbursts occurs, $j$ may reach fairly large negative values. For instance, Fig. 4 shows that $j=-1$ for $\Omega_{*} \simeq 1.83 \times 10^{-5} \mathrm{~s}^{-1}$, which is only about a $6 \%$ increase in $\Omega_{*} / \Omega_{K}\left(R_{*}\right)$ over the point where $j=0$. A $10 \%$ variation from $\Omega_{*} / \Omega_{K}\left(R_{*}\right) \simeq 0.3$ to 0.4 , as discussed above, would give $j \sim-2$. This would give an accretion luminosity of $\sim 1.88 G M_{*} \dot{M} / R_{*}$, which would make the outburst even brighter than one would expect from the increase in $\dot{M}$. This additional luminosity would come from the rotational energy of the star, which is released as the star spins down.

Disk solutions with negative angular momentum accretion rates may be important in other types of accreting systems. In systems such as cataclysmic variables, the accretion rates are generally much smaller than those of FU Orionis systems. This produces a narrower boundary layer, and pressure support plays a smaller role, so we would expect that negative angular momentum accretion rates are only found for $\Omega_{*} \simeq \Omega_{K}\left(R_{*}\right)$. We are currently completing a study of negative- $j$ solutions for disks around cataclysmic variables with $\dot{M}=10^{-8} \mathrm{M}_{\odot} \mathrm{yr}^{-1}$ which confirms these expectations (Popham 1995). In some other systems, such as embedded pre-main-sequence stars, or some X-ray binaries, the accretion rates may be large enough to produce solutions like the ones presented here, where negative angular momentum accretion rates are reached when the star is still spinning relatively slowly.

\subsection{Assumptions}

The results described above depend on a number of assumptions. One very important assumption is that FU Orionis outbursts occur in classical T Tauri stars; i.e., that the T Tauri and FU Orionis phases occur during the same epoch of pre-main sequence stellar evolution. Models of FU Orionis outbursts based on disk instabilities generally assume that the outbursts arise in $\mathrm{T}$ Tauri star disks, so that the FU Orionis and $\mathrm{T}$ Tauri phases correspond to the high and low states observed in other types of accreting stars, such as dwarf novae. The observational connection between T Tauri and FU Orionis stars is rather tenuous, since only one of the current FU Orionis systems was observed spectroscopically before it went into outburst, and no known FU Orionis systems have yet returned to their 
pre-outburst levels. The one system for which a spectrum was taken prior to outburst, V1057 Cygni, resembled a typical T Tauri star (Herbig 1977). However, some of the observed characteristics of FU Orionis systems, such as their association with reflection nebulae and outflows, and their large far-infrared excesses, suggest that FU Orionis outbursts may arise in younger embedded sources rather than in classical T Tauri stars (Kenyon 1995). If this is the case, then the mechanism described here should serve to limit the stellar rotation rate during the embedded phase of pre-main-sequence stellar evolution. The rotation rate would then presumably be controlled by the stellar magnetic field during the T Tauri phase.

We must also make some assumption about the location of the stellar radius, which is difficult to define in our model (see $\S 4$ for a discussion of this problem). We have chosen a particular value of $v_{R, *}$ to define $R_{*}$, in order to provide an example of the variation of $H_{*}$ and $j$ as a function of $\Omega_{*}$. Unfortunately, the value of $\Omega_{*}(j=0)$ is fairly sensitive to the choice of $v_{R, *}$. We have illustrated this in Fig. 4, where we have plotted lines corresponding to $v_{R, *}=-500 \mathrm{~cm} \mathrm{~s}^{-1}$ and $v_{R, *}=-2000 \mathrm{~cm} \mathrm{~s}^{-1}$. For these values of $v_{R, *}, j$ reaches zero at $\Omega_{*} \simeq 0.94$ and $2.19 \times 10^{-5} \mathrm{~s}^{-1}$, respectively. This means that for a factor of 4 variation in $v_{R, *}, \Omega_{*}(j=0)$ varies by more than a factor of 2 . Thus, even when we specify all the parameters of an FU Orionis system, we still have a substantial uncertainty in $\Omega_{*}(j=0)$ resulting from the uncertainty in the definition of the stellar radius. As discussed in $\S 4.5$, $\Omega_{*}(j=0)$ also varies dramatically with $\dot{M}$. Therefore we can only say that for what we believe to be reasonable choices of $v_{R, *}$ and $\dot{M}$, the resulting values of $\Omega_{*}(j=0)$ are comparable to $\mathrm{T}$ Tauri rotation rates.

Our results are not strongly dependent on the magnetic field of the star, as long as the field is not strong enough to disrupt the accretion flow in the high- $\dot{M}$ FU Orionis phase. This would require a field substantially larger than the $\sim 1 \mathrm{kG}$ needed to disrupt the disk during the $\mathrm{T}$ Tauri phase. The disruption radius varies as $R_{d} \propto \dot{M}^{-2 / 7} B^{4 / 7}$ (Pringle \& Rees 1972), so to maintain the same $R_{d}$ requires that $B$ vary as $\dot{M}^{1 / 2}$. Since FU Orionis accretion rates are a factor of $\sim 1000$ higher than T Tauri rates, $B$ would have to be about 30 times larger to disrupt the disk, which would require $B \sim 3 \times 10^{4} \mathrm{G}$. A field this strong would disrupt $\mathrm{T}$ Tauri disks at $R \gtrsim 20 R_{*}$, which can probably be ruled out by observations.

We have used stellar radii of $2,25,3$, and $4 \times 10^{11} \mathrm{~cm}$, corresponding to $2.88,4.31$, and $5.75 \mathrm{R}_{\odot}$, in our calculations. These are larger than the radii generally found for $\mathrm{T}$ Tauri stars, which are in the range $1.5-2.5 \mathrm{R}_{\odot}$. These larger radii appear to be required by observations: smaller stellar radii would produce maximum temperatures in the inner disk which are substantially larger than those observed. This suggests that the accreting star may expand during the FU Orionis outburst. 
Prialnik \& Livio (1985) calculated the evolution of a fully convective $0.2 \mathrm{M}_{\odot}$ star during the accretion of $2.5 \times 10^{-3} \mathrm{M}_{\odot}$ of material at rates ranging from $10^{-1}$ to $10^{-10} \mathrm{M}_{\odot} \mathrm{yr}^{-1}$. They also varied the fraction $f$ of the accretion energy carried into the star with the accreting material; $f$ ranged from 0.001 to 0.5 . They found that for the accretion rates appropriate to FU Orionis outbursts, the star expanded stably if $f \leq 0.03$. If $f \geq 0.1$, the star expanded unstably. In our FU Orionis solutions, the central temperature of the disk at $R_{*}$ is $\sim 1-3 \times 10^{5} \mathrm{~K}$. The virial temperature for these parameters is $\sim 10^{6} \mathrm{~K}$, so a reasonable fraction $f \sim 0.1-0.3$ of the accretion energy is advected into the star. This suggests that rapid stellar expansion may result from the high- $\dot{M}$ accretion during an FU Orionis outburst, but it is difficult to know how the mass of the star, the presence of rotation, and the departure from spherical symmetry would affect the Prialnik \& Livio results. This expansion, and the subsequent contraction during the $\mathrm{T}$ Tauri phase, could affect the spin evolution of the star, and other aspects of the star's interaction with the disk, and clearly deserves further study.

Our models also assume that the disk and boundary layer are in a steady state. This is clearly not true for FU Orionis systems, since they are experiencing outbursts. The viscous timescale is $t_{v i s c} \simeq R^{2} / \nu=\alpha^{-1}(H / R)^{-2} \Omega_{K}^{-1}$. For our solutions with $\alpha=0.01$,

$H / R \simeq 0.3$, this gives $t_{v i s c} \simeq 1000 \Omega_{K}^{-1}$. In the inner disk, we have $\Omega_{K} \simeq$ few $\times 10^{-5} \mathrm{~s}^{-1}$, so $t_{\text {visc }} \simeq$ few $\times 10^{7} \mathrm{~s} \simeq 1 \mathrm{yr}$. This is comparable to the rise times of the fastest-rising systems, FU Ori and V1057 Cyg, and much shorter than their decline times. Thus FU Orionis systems should be reasonably well-modeled by steady state solutions for this value of $\alpha$.

\subsection{Comparison with Observations}

Recent measurements of periodic photometric variations in $\mathrm{T}$ Tauri stars have clearly established that the classical disk-accreting $\mathrm{T}$ Tauri stars spin more slowly than weak-line T Tauri stars, which appear to lack accretion disks (Bouvier et al. 1993, 1995; Edwards et al. 1993). This has generally been interpreted to mean that the spinup of $\mathrm{T}$ Tauri stars is controlled by a stellar magnetic field strong enough to disrupt the disk. If FU Orionis outbursts regulate the spin evolution of $\mathrm{T}$ Tauri stars in the way described in this paper, it would also account for this observation. In both cases, disk accretion maintains the slow rotation speed of classical T Tauri stars. If FU Orionis outbursts cease late in the classical $\mathrm{T}$ Tauri stage, then the more rapid rotation of the weak-line $\mathrm{T}$ Tauri stars could be produced by accretion at the end of the classical T Tauri stage.

Observations of the spin evolution of T Tauri stars could be used to test the ideas discussed in this paper. The most useful data would be measurements of the spin period 
before and after an FU Orionis outburst. If the star spins down during an outburst, it would offer clear evidence that outbursts are regulating the rotation rates of $\mathrm{T}$ Tauri stars. If the star spins up, the result is less clear. If the amount of spinup is less than expected based on the amount of mass accreted during the outburst, it would suggest that the star is reaching the equilibrium rotation rate $\Omega_{*, e q, F U}$ at some point during the outburst. In this case the outbursts would still be limiting the rotation rate of the star. If, on the other hand, the star spins up as much as expected, it would suggest that the rotation rate must be limited by processes taking place during the $\mathrm{T}$ Tauri phase. One complication in interpreting the observations is the possibility that significant amounts of angular momentum might be carried off in the strong outflows which occur during outbursts.

Observations of variations in the rotation rates of $\mathrm{T}$ Tauri stars which have not experienced observed outbursts could also be useful. Over a short timescale, variations in $\dot{M}$ could produce irregular period variations. Nonetheless, if FU Orionis outbursts are controlling the rotation rates of these stars, then their spin periods should vary secularly during the $\mathrm{T}$ Tauri phase as the rotation rate $\Omega_{*}$ moves from $\Omega_{*, e q, F U}$ toward $\Omega_{*, e q, T T}$. If instead the rotation rates of $\mathrm{T}$ Tauri stars remain constant over a long timescale, it would suggest that $\Omega_{*}$ has stabilized at $\Omega_{*, e q, T T}$, which would indicate that the spin evolution is being controlled by angular momentum transfer in the T Tauri phase.

The main sources of data which can be used to further constrain the solutions presented in this paper are the spectra of FU Orionis systems. These spectra yield valuable information on the disk temperatures and rotational velocities (Hartmann \& Kenyon 1985, 1987; KHH). The solutions presented in this paper were selected to have luminosities and temperatures in approximate agreement with those derived from observations. Because our main aim in this paper has been to understand the spinup and angular momentum transfer in FU Orionis systems, we have not attempted to match our solutions with observations in any detail. We defer this to a subsequent paper (Popham et al. 1995), where we plan to compare the spectra and rotational velocities produced by our disk and boundary layer solutions to those observed in FU Orionis systems.

I would like to thank Ramesh Narayan, Scott Kenyon, and Lee Hartmann for helpful discussions. I would also like to thank the organizers of the conference on Circumstellar Disks, Outflows and Star Formation, held in Cozumel, Mexico, where an early version of this work was presented. This work was supported by NASA grant NAG5-2837 at the Center for Astrophysics and grants NASA NAGW-1583, NSF AST 93-15133, and NSF PHY 91-00283 at the University of Illinois. 


\section{REFERENCES}

Bell, K. R., \& Lin, D. N. C. 1994, ApJ, 427, 987.

Bell, K. R., Lin, D. N. C., Hartmann, L. W., \& Kenyon, S. J. 1995, ApJ, 444, 376.

Bouvier, J., Cabrit, S., Fernandez, M., Martin, E. L., \& Matthews, J. M. 1993, A\&A, 272, 176.

Bouvier, J., Covino, E., Kovo, O., Martin, E. L., Matthews, J. M., Terranegra, L., \& Beck, S. C. 1995, A\&A, 299, 89.

Cameron, A. C., \& Campbell, C. G. 1993, A\&A, 274, 309.

Clarke, C. J., Lin, D. N. C., \& Papaloizou, J. C. B. 1989, MNRAS, 236, 495.

Clarke, C. J., Lin, D. N. C., \& Pringle, J. E. 1989, MNRAS, 242, 439.

Edwards, S., et al. 1993, AJ, 106, 372.

Ghosh, P., Lamb, F. K., \& Pethick, C. J. 1977, ApJ, 217, 578.

Ghosh, P., \& Lamb, F. K. 1979a, ApJ, 232, 259.

Ghosh, P., \& Lamb, F. K. 1979b, ApJ, 234, 296.

Hartmann, L. 1994, in Theory of Accretion Disks 2, ed. W. J. Duschl et al. (Dordrecht: Kluwer), p. 19.

Hartmann, L., \& Kenyon, S. J. 1985, ApJ, 299, 462.

Hartmann, L., \& Kenyon, S. J. 1987, ApJ, 312, 243.

Hartmann, L., Kenyon, S. J., \& Hartigan, P. 1993, in Protostars and Planets III, ed. E. H. Levy \& J. I. Lunine (Tucson: Univ. of Arizona Press), 497.

Herbig, G. H. 1977, ApJ, 217, 693.

Hubeny, I. 1990, ApJ, 351, 632.

Kenyon, S. J. 1995, Rev. Mex. A. A. (Serie de Conferencias), 1, 237.

Kenyon, S. J., Hartmann, L., \& Hewett, R. 1988, ApJ, 325, 231 (KHH).

Kenyon, S. J., Hartmann, L., Imhoff, C. L., \& Cassatella, A. 1989, ApJ, 344, 925.

Königl, A. 1991, ApJ, 370, L39.

Muchotrzeb, B., \& Paczyński, B. 1982, Acta Astron., 32, 1.

Narayan, R., \& Popham, R. 1993, Nature, 362, 820.

Paczyński, B., \& Bisnovatyi-Kogan, B. 1981, Acta Astron., 31, 283.

Popham, R., \& Narayan, R. 1991, ApJ, 370, 604 (PN91). 
Popham, R. 1995, in preparation.

Popham, R., \& Narayan, R. 1995, ApJ, 442, 337.

Popham, R., Narayan, R., Hartmann, L., \& Kenyon, S. 1993, ApJ, 415, L127 (PNHK).

Popham, R., Narayan, R., Hartmann, L., \& Kenyon, S. 1995, in preparation.

Prialnik, D., \& Livio, M. 1985, MNRAS, 216, 37.

Pringle, J. E., \& Rees, M. J. 1972, A\&A, 21, 1.

Shakura, N. I., \& Sunyaev, R. A. 1973, A\&A, 24, 337.

Shu, F. H., Najita, J., Ostriker, E., Wilkin, F., Ruden, S., \& Lizano, S. 1994, ApJ, 429, 781. 
Fig. 1.- A boundary layer solution for typical FU Orionis parameters. The solution shown here has $\dot{M}=10^{-4.15} \mathrm{M}_{\odot} \mathrm{yr}^{-1}, M_{*}=0.5 \mathrm{M}_{\odot}, R_{*}=3 \times 10^{11} \mathrm{~cm}, j=0.90, H_{*}=10^{11} \mathrm{~cm}$, and $\alpha=10^{-2}$. The four panels show the angular velocity $\Omega$, the radial velocity $v_{R}$, the effective temperature $T_{e f f}$, and the disk height $H$ in the range from $R=3-9 \times 10^{11} \mathrm{~cm}=1-3 R_{*}$. The dashed line shows the Keplerian angular velocity $\Omega_{K}$.

Fig. 2.- Variation of the rotation rate $\Omega_{*}$ and the disk height $H_{*}$ at $R=R_{*}=3 \times 10^{11} \mathrm{~cm}$, for solutions with $j=0.90, \dot{M}=10^{-4.15} \mathrm{M}_{\odot} \mathrm{yr}^{-1}, M_{*}=0.5 \mathrm{M}_{\odot}, R_{*}=3 \times 10^{11} \mathrm{~cm}$, and $\alpha=10^{-2}$. The solutions form a Z-shaped curve in the $\Omega_{*}-H_{*}$ plane, with three solution branches.

Fig. 3.- Same as Fig. 1, but for the 10 solutions marked along the Z-shaped curve in Fig. 2 , with $H_{*}=0.80,0.85,0.90, \ldots 1.25 \times 10^{11} \mathrm{~cm}$. The boundary layer width increases rapidly as $H_{*}$ increases, and angular velocity, radial velocity, and effective temperature all decrease.

Fig. 4.- Same as Fig. 2, but for various values of $j$ as labeled. All of the solutions on all curves have $\dot{M}=10^{-4.15} \mathrm{M}_{\odot} \mathrm{yr}^{-1}, M_{*}=0.5 \mathrm{M}_{\odot}, R_{*}=3 \times 10^{11} \mathrm{~cm}$, and $\alpha=10^{-2}$. As $\Omega_{*}$ and $H_{*}$ increase, $j$ decreases, and the curves change from Z-shaped to monotonic. The dotted line marks the solutions with $v_{R, *}=-1000 \mathrm{~cm} \mathrm{~s}^{-1}$, the upper dashed line $v_{R, *}=-500 \mathrm{~cm} \mathrm{~s}^{-1}$, the lower dashed line $v_{R, *}=-2000 \mathrm{~cm} \mathrm{~s}^{-1}$.

Fig. 5.- A boundary layer solution with $j=0$, where the star accretes mass but no angular momentum. This solution has $\dot{M}=10^{-4.15} \mathrm{M}_{\odot} \mathrm{yr}^{-1}, M_{*}=0.5 \mathrm{M}_{\odot}, R_{*}=3 \times 10^{11} \mathrm{~cm}$, $H_{*} \simeq 1.08 \times 10^{11} \mathrm{~cm}$, and $\alpha=10^{-2}$. Note that $v_{R, *}=-1000 \mathrm{~cm} \mathrm{~s}^{-1}$, as discussed in $\S 4.4$; if this condition is used to define $R_{*}$, then $j=0$ for $\Omega_{*} \simeq 1.55 \times 10^{-5} \mathrm{~s}^{-1}$, corresponding to a rotation period of 4.7 days.

Fig. 6. - Same as Fig. 4, but for two other choices of $\dot{M}$ and $R_{*}$ : (a) has $\dot{M}=$ $10^{-4.0} \mathrm{M}_{\odot} \mathrm{yr}^{-1}$ and $R_{*}=4 \times 10^{11} \mathrm{~cm}=5.75 \mathrm{R}_{\odot}$, and $(\mathrm{b})$ has $\dot{M}=10^{-4.3} \mathrm{M}_{\odot} \mathrm{yr}^{-1}$ and $R_{*}=2.25 \times 10^{11} \mathrm{~cm}=3.23 \mathrm{R}_{\odot}$. Note that as $\dot{M}$ increases, the Z-shape of the curves becomes less pronounced and only appears at lower values of $\Omega_{*}$. The dotted lines mark the solutions with $v_{R, *}=-1000 \mathrm{~cm} \mathrm{~s}^{-1}$.

Fig. 7.- Variation of the angular momentum accretion rate $j$ with the stellar rotation rate $\Omega_{*}$, for a set of solutions with a given value of $v_{R}$ at $R_{*}$ : (a) is for $\dot{M}=10^{-4.15} \mathrm{M}_{\odot} \mathrm{yr}^{-1}$, $R_{*}=3 \times 10^{11} \mathrm{~cm}$, and shows $j\left(\Omega_{*}\right)$ for $v_{R, *}=-500,-1000$, and $-2000 \mathrm{~cm} \mathrm{~s}^{-1} ;$ (b) is for $\dot{M}=10^{-4.0} \mathrm{M}_{\odot} \mathrm{yr}^{-1}, R_{*}=4 \times 10^{11} \mathrm{~cm}$, and $v_{R, *}=-1000 \mathrm{~cm} \mathrm{~s}^{-1}$; and (c) is for $\dot{M}=10^{-4.3} \mathrm{M}_{\odot} \mathrm{yr}^{-1}, R_{*}=2.25 \times 10^{11} \mathrm{~cm}$, and $v_{R, *}=-1000 \mathrm{~cm} \mathrm{~s}^{-1}$. In all cases, $j$ drops with increasing $\Omega_{*}$ and reaches $j=0$ for $\Omega_{*}$ well below $\Omega_{K}\left(R_{*}\right)$. 

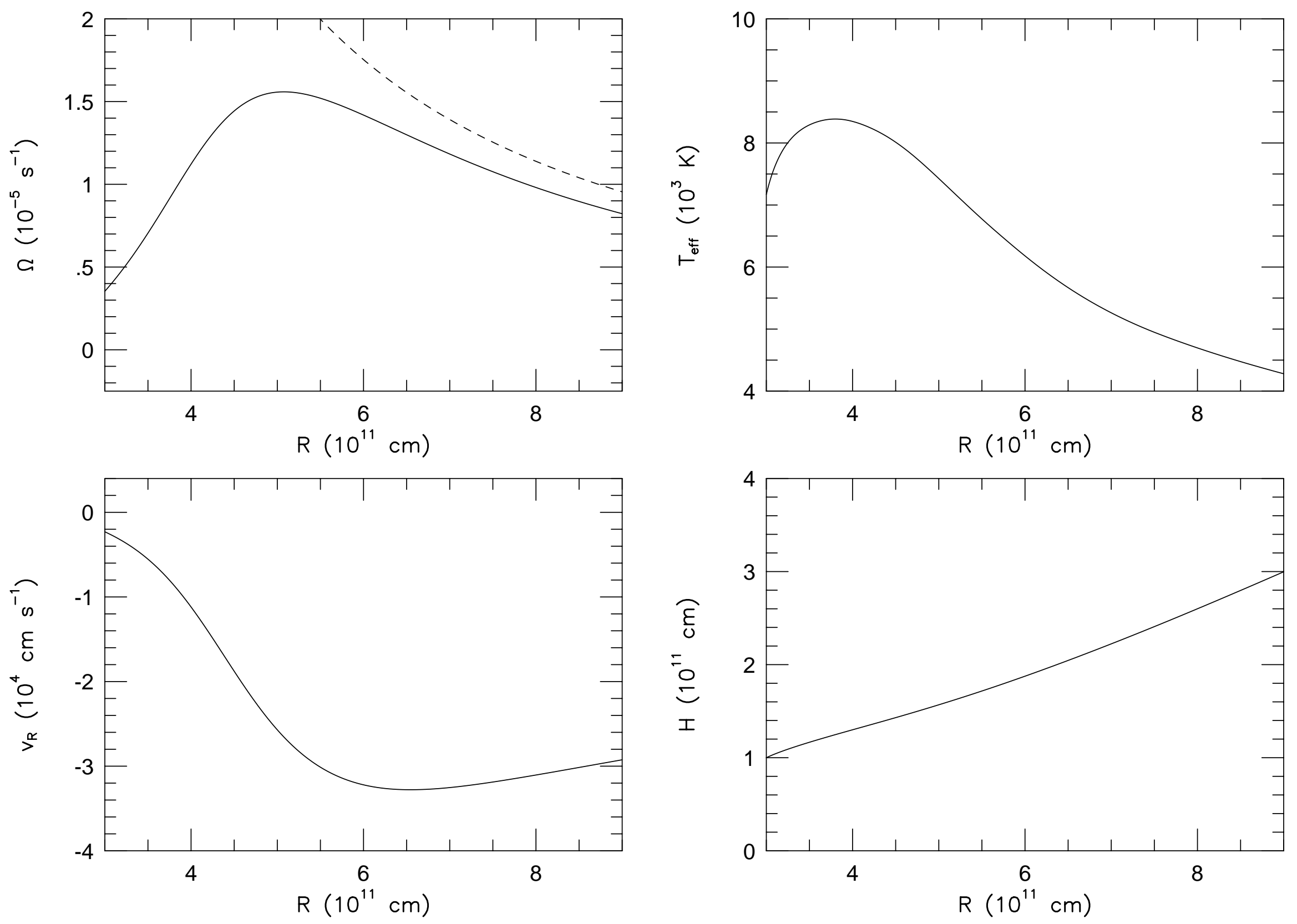


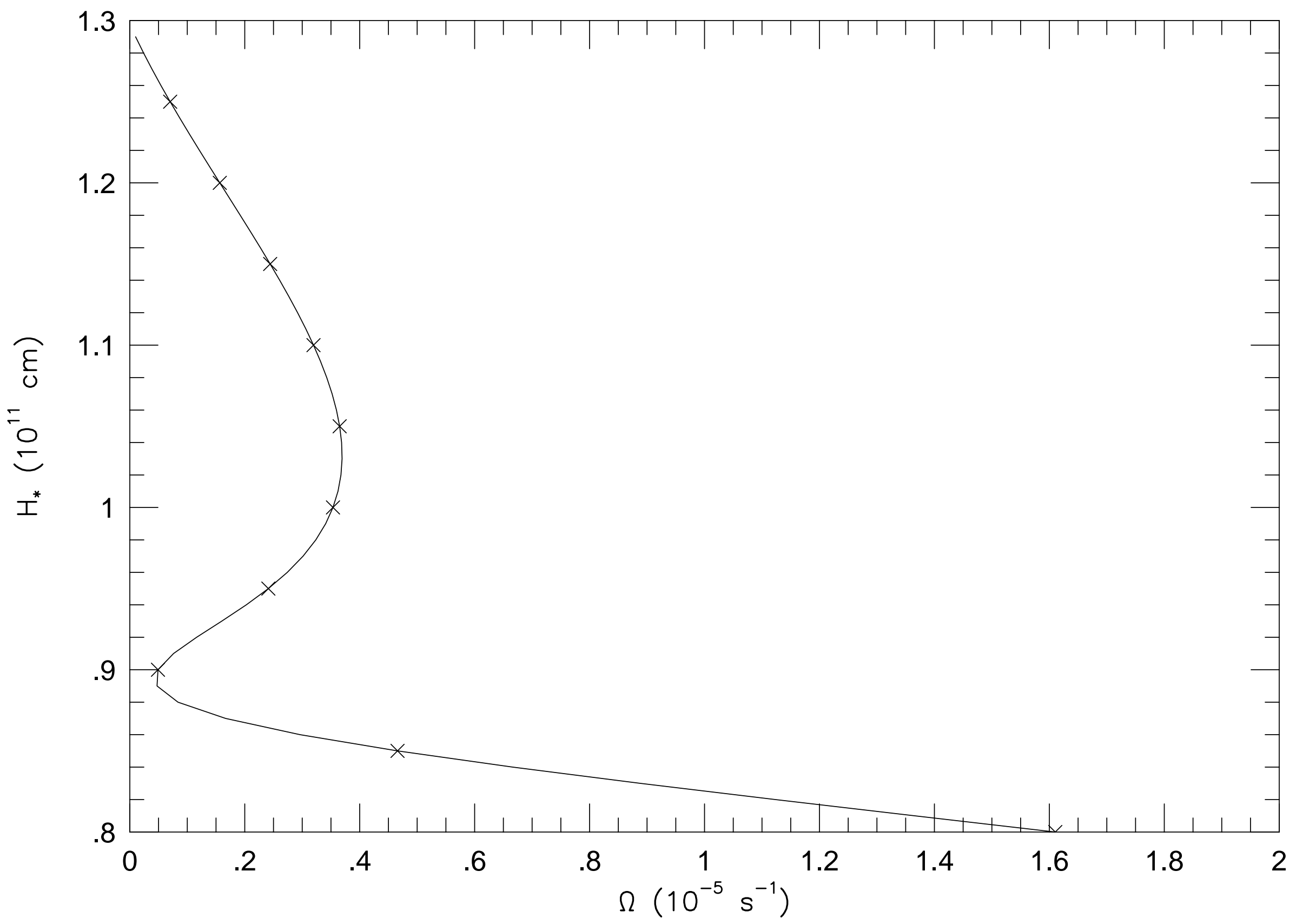



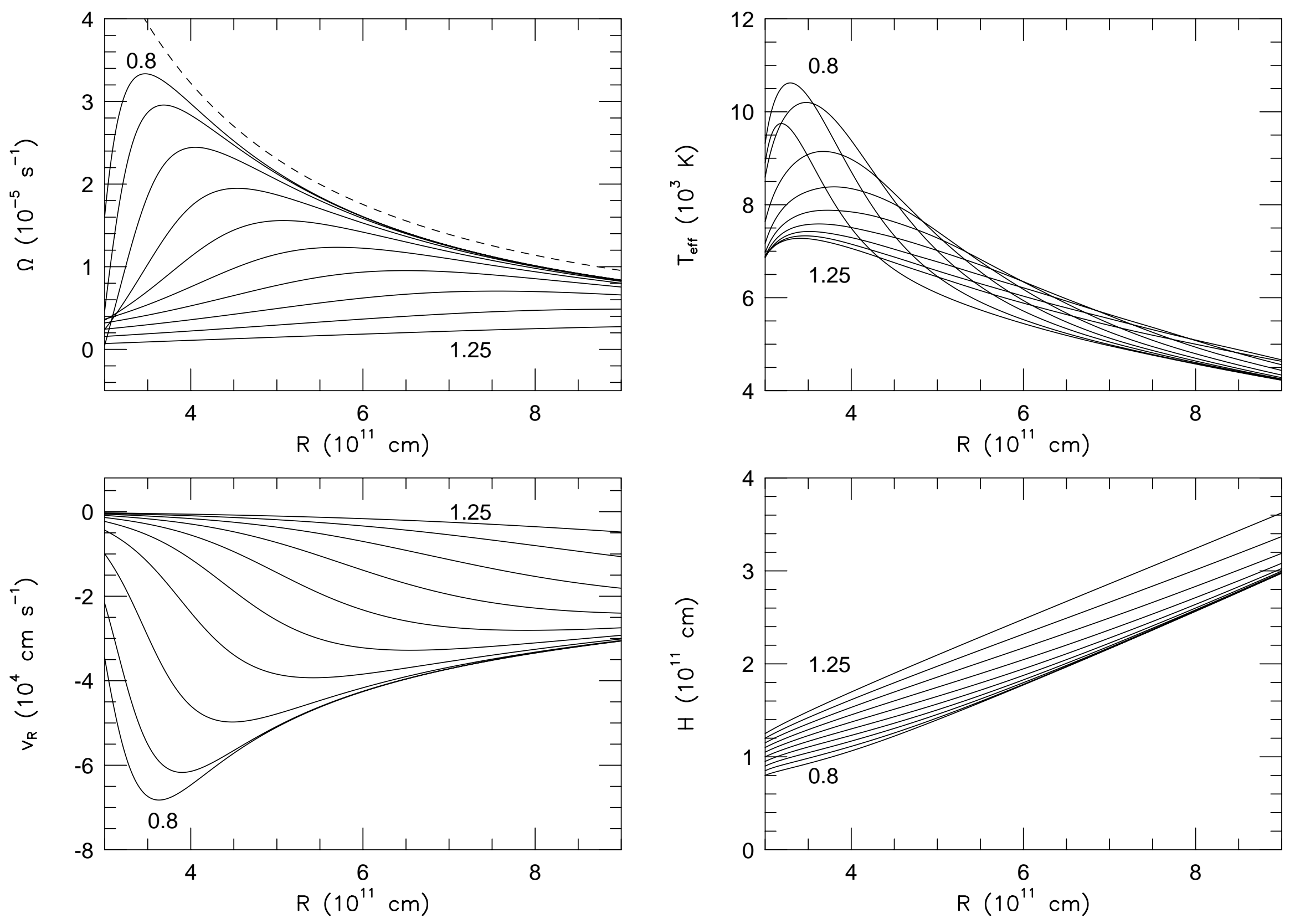


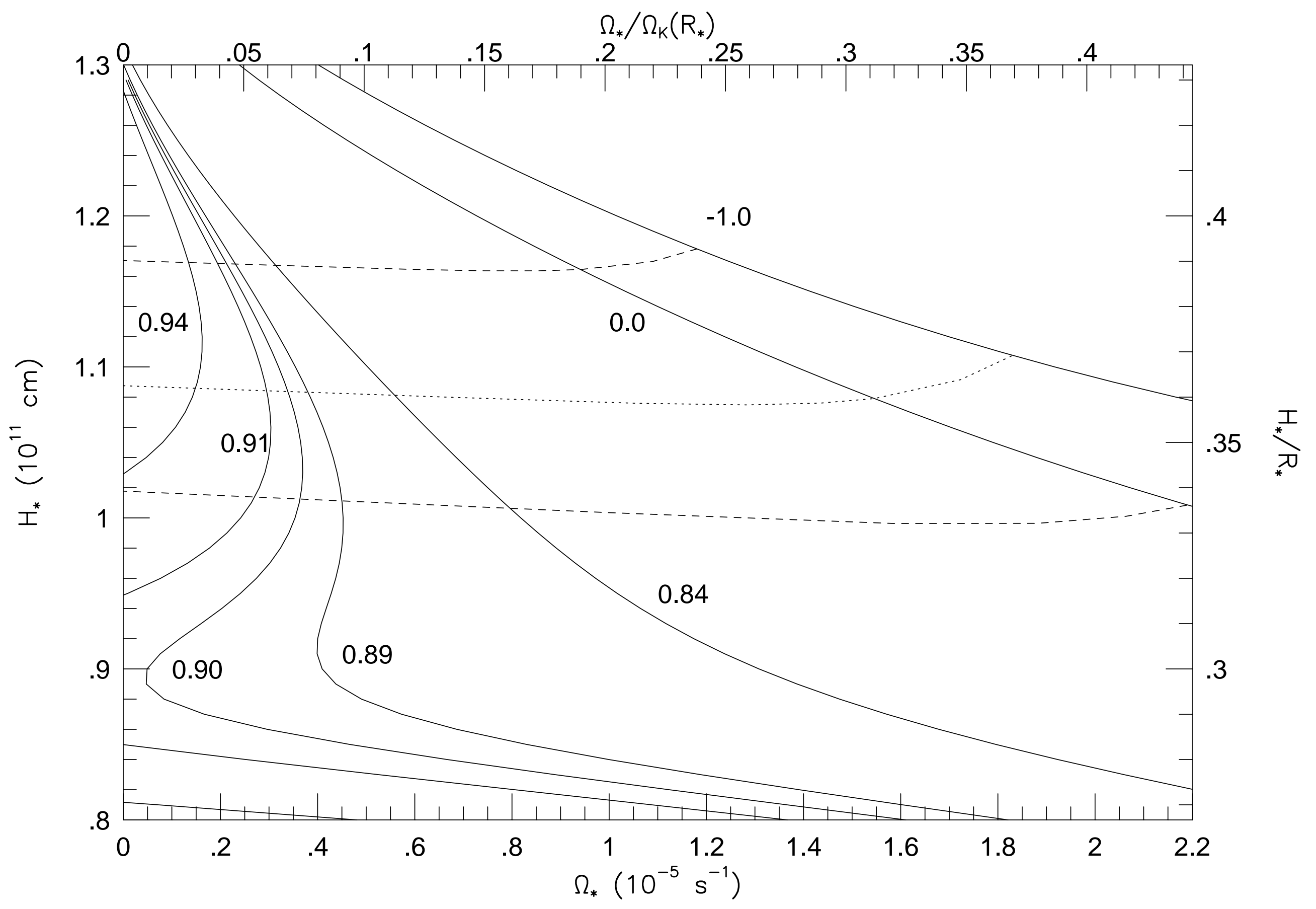



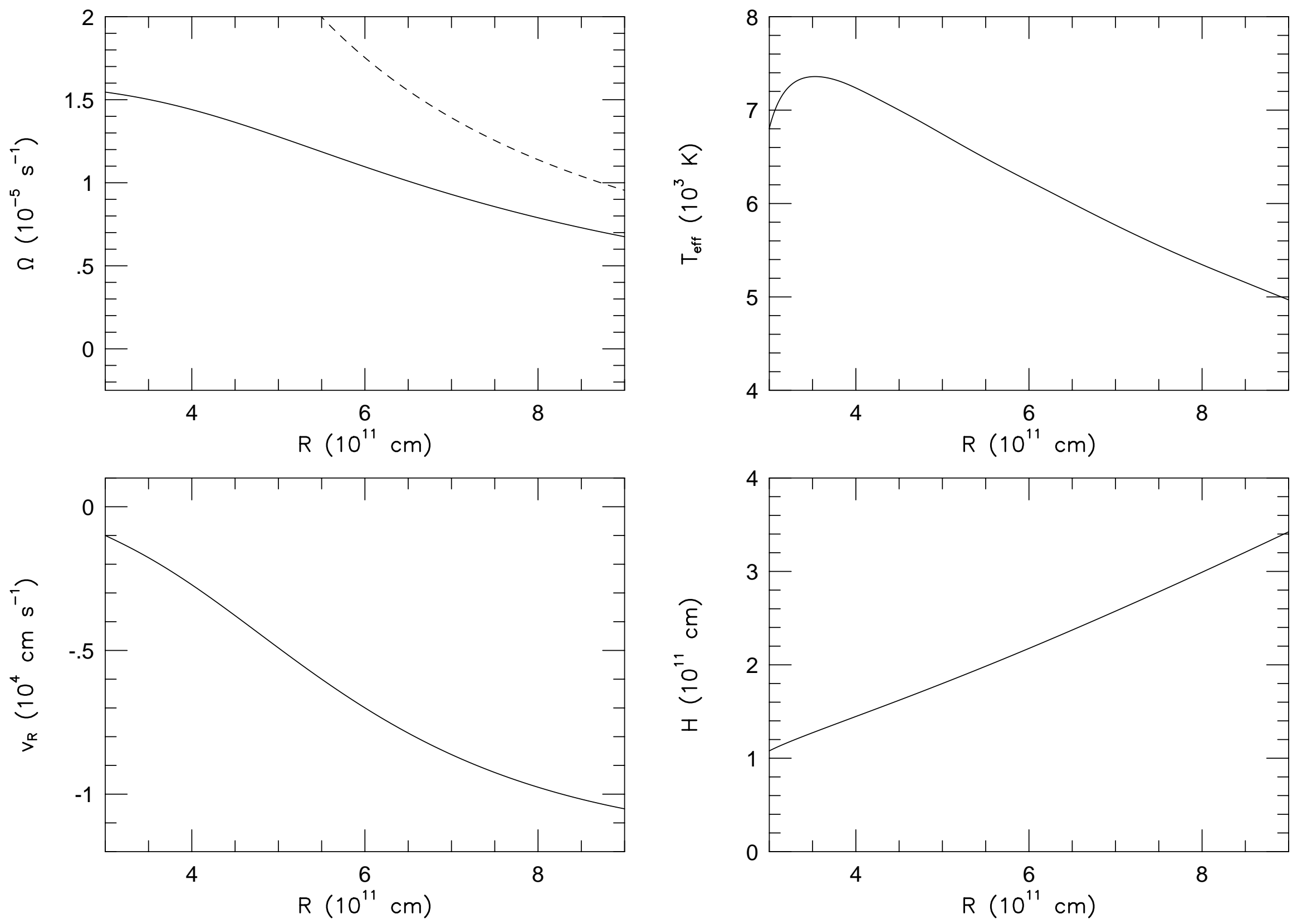

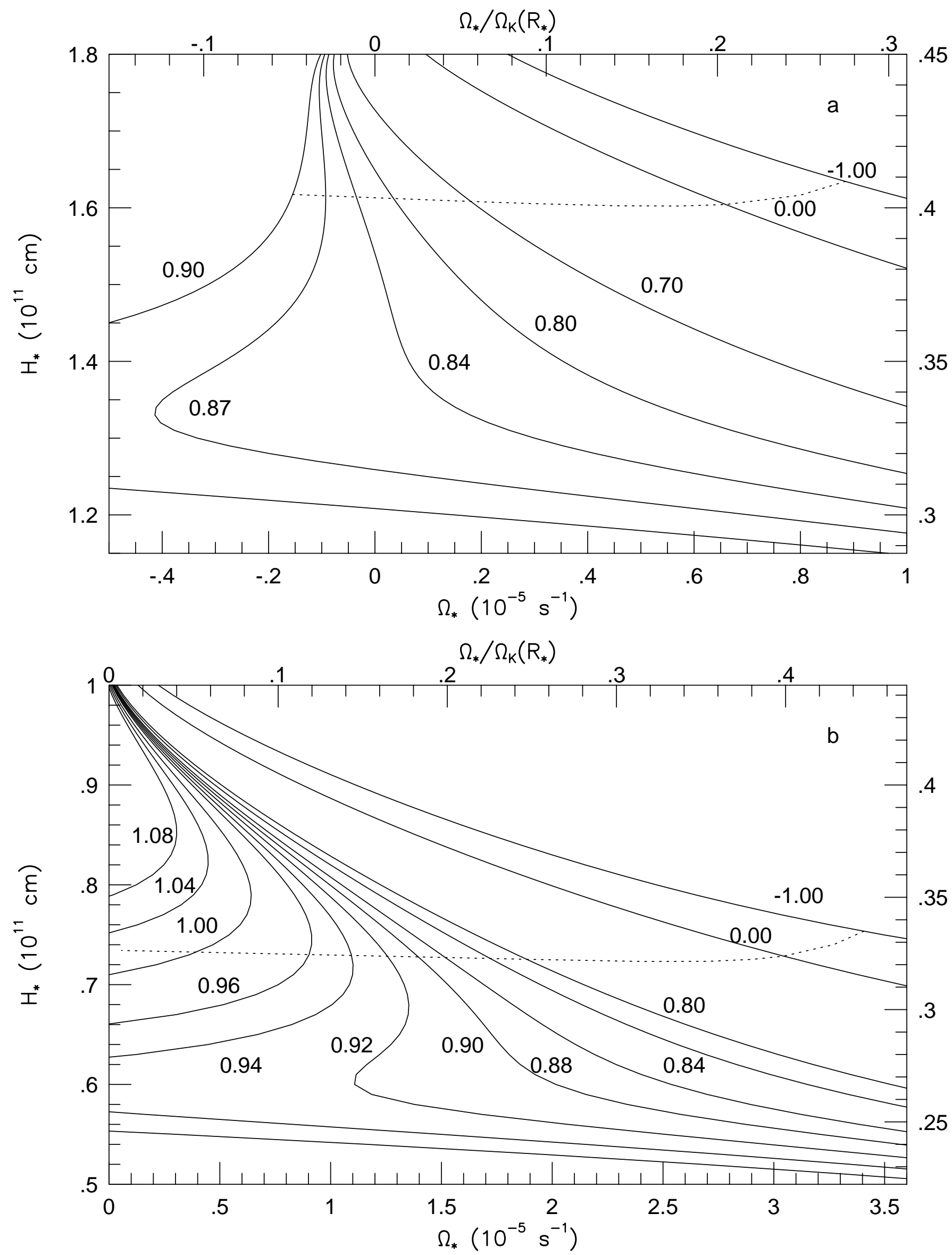

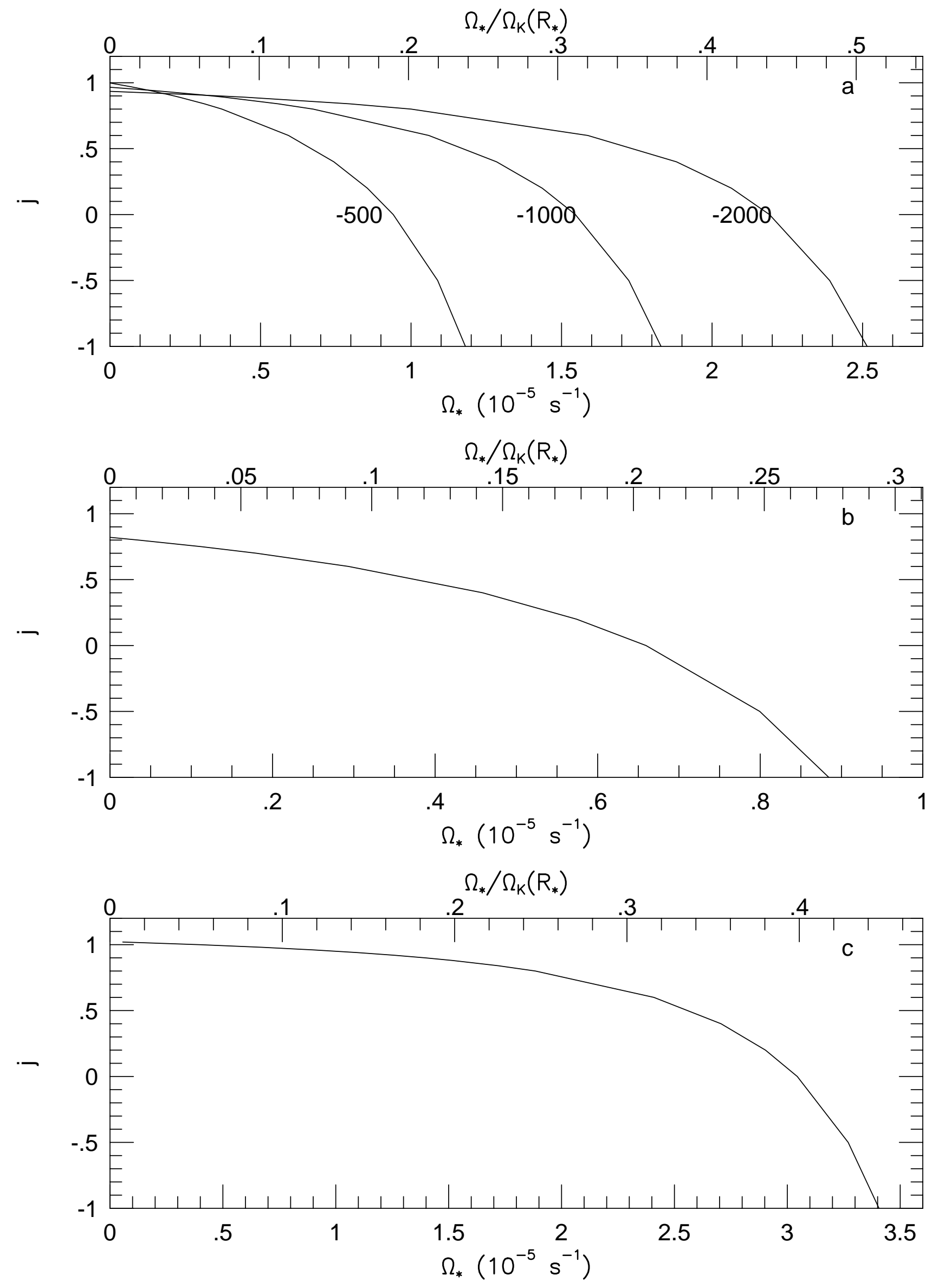